\title{
La manipulación de las masas en los Estados Unidos del periodo de entreguerras (1919-1941): investigaciones legislativas como respuesta a la inquietud popular
}

\author{
Dario Migliucci ${ }^{1}$
}

Recibido: 16 de diciembre de 2019 / Aceptado: 4 de septiembre de 2020

Resumen. Durante el periodo de entreguerras se difundió en los Estados Unidos una profunda inquietud por las actividades de manipulación de la opinión pública. Ante la imposibilidad de aplicar medidas coercitivas concretas -ya que hubiesen sido inconstitucionales- el Congreso emprendió numerosas investigaciones legislativas sobre la labor propagandística de grupos subversivos, corporaciones privadas y agencias gubernamentales. El presente trabajo pretende reconstruir el clima en el que dichos comités fueron instituidos, analizando la forma en la que las ansiedades populares de aquel periodo influyeron en su modus operandi y en los resultados que presentaron ante las cámaras legislativas y los medios de comunicación.

Palabras clave: Propaganda; opinión pública; periodo de entreguerras, comités legislativos de investigación, Congreso de los Estados Unidos de América; siglo XX.

\section{[en] Manipulation of the Masses in the United States during the Interwar Period (1919-1941): Legislative Research as a Response to Popular Concerns}

\begin{abstract}
During the interwar period, a deep concern spread across the United States regarding activities of manipulation of public opinion. Faced with the impossibility of applying certain coercive measures that would have been unconstitutional, Congress undertook numerous legislative investigations of the propagandistic activities of subversive groups, private corporations and government agencies. This paper proposes to reconstruct the climate in which these committees were instituted, by analyzing the manner in which the popular anxieties of the period influenced the committees' modus operandi and the results presented before the legislative chambers and the communications media.

Keywords: Propaganda; Public Opinion; Interwar Period; Legislative Investigation Committees; Congress of the United States of America; 20th Century.
\end{abstract}

Sumario. 1. Introducción. 2. La propaganda radical durante la primera posguerra. 3. La propaganda de las grandes empresas. 4. Viejos y nuevos enemigos: la propaganda antiamericana. 5. La política y la propaganda de la política. 6 . Conclusiones. 7. Referencias bibliográficas.

Cómo citar: Migliucci, D. (2021) La manipulación de las masas en los Estados Unidos del periodo de entreguerras (1919-1941): investigaciones legislativas como respuesta a la inquietud popular, en Revista Complutense de Historia de América 47, 233-253.

1 Investigador posdoctoral (convocatoria 2020) en la Universidad Nacional Autónoma de México. La investigación que ha llevado a la realización del artículo, sin embargo, fue financiada por el Ministerio de Educación del Gobierno de España a través de una ayuda FPU (FPU14/01884).

E-mail: dariomigliucci@hotmail.com 


\section{Introducción}

La de la propaganda fue sin duda una las grandes problemáticas que caracterizaron la acción política y el debate intelectual en la mayoría de las naciones democráticas del periodo de entreguerras. El fenómeno de la manipulación de consumidores y electores no era por supuesto ninguna novedad. En países como los Estados Unidos de América, diversas polémicas relacionadas con el abuso de campañas publicitarias habían involucrado -a caballo entre los siglos XIX y XX-tanto a empresas privadas como a partidos y representantes del mundo institucional ${ }^{2}$. Sin embargo, la inquietud por la manipulación de las masas se disparó tras el fin de la Primera Guerra Mundial, cuando -precisamente debido a las circunstancias bélicas y a la realidad política de la posguerra- el concepto de "propaganda" comenzó a asumir nuevos e inquietantes matices ${ }^{3}$. Un papel transcendental, a la hora de crear una sensación de desamparo e intranquilidad ante las diferentes estrategias de persuasión, lo habían jugado los aparatos institucionales de propaganda que fueron establecidos durante el conflicto por los países beligerantes ${ }^{4}$. Dichas estructuras, instituidas por el poder ejecutivo y financiadas con dinero público, habían demostrado ser extremadamente eficaces a la hora de moldear opiniones y actitudes de la ciudadanía. El miedo a que esas estrategias de manipulación pudieran ser retomadas en tiempo de paz por empresarios y dirigentes políticos o que movimientos totalitarios pudieran establecer aparatos de persuasión permanentes, hizo que se intensificara el temor a la manipulación por la propaganda.

Los Estados Unidos fueron uno de los países en los que más manifiestas se hicieron estas preocupaciones. Eso se debió en gran medida a los debates que se originaron en el mundo intelectual estadounidense de la posguerra sobre el impacto que tuvieron, en la opinión pública norteamericana, las actividades del Committee on Public Information, uno de los aparatos de propaganda más imponentes de la Gran Guerra. Lo cierto es que, en muy pocos meses, las campañas informativas de dicho comité lograron convencer de la bondad de la intervención militar estadounidense a una población que hasta entonces se había mostrado decididamente reacia a la guerra. Salvo destacadas excepciones, la población norteamericana se compactó alrededor de los intereses nacionales. Los jóvenes se alistaron con gran espíritu patriótico, el ciudadano medio se volcó en la compra de Liberty Bonds y la xenofobia anti-alemana creció considerablemente, demostrándose así de forma incuestionable el enorme poder de la persuasión masiva llevada a cabo por expertos en técnicas de control de la información ${ }^{5}$.

El panorama internacional de aquellos años también contribuyó a crear una grave sensación de intranquilidad. En Europa diversos movimientos radicales se hicieron con el poder, lo que finalmente resultó en la aparición de regímenes totalitarios en naciones como Rusia, Italia o Alemania. Los estadounidenses, todavía tocados por los desafíos anarquista y sindicalista que hostigaban al país desde hacía varias déca-

2 Muchas de estas polémicas se debieron a la actividad de los llamados muckrakers, periodistas que se dedicaron a denunciar escándalos políticos y financieros. Véase Sproule, 1997: 23-29.

3 Sobre las nuevas connotaciones que se dieron a conceptos como "propaganda" y "opinión pública" en los primeros años de paz, véase Migliucci, 2018a.

4 Audoin-Rouzeau, 1990; Axelrod, 2009; Hanna, 1996; Kaminski, 2014; Lasswell, 1927; Marquis, 1978; Messinger, 1992; Mock - Larson, 1939; Row, 2002; Sanders - Taylor, 1982; Vaughn, 1980.

5 El Committee on Public Information contrató a un gran número de periodistas y expertos en publicidad. Véase Creel, 1920. 
das, tras el fin de la guerra comenzaron a percibir las amenazas de los movimientos bolchevique y fascista. También provocó gran recelo, a partir de los años veinte, el desmesurado crecimiento de un modelo económico asentado en el consumo de masas. Las técnicas publicitarias y el desarrollo de las relaciones públicas se convirtieron en los grandes ejes de un sistema que aseguraba sus ventas a través de eficaces campañas de persuasión. Muchos observadores de la época manifestaron inquietud ante la posibilidad de que los consumidores pudiesen ser embaucados por anuncios fraudulentos, sobre todo después de la popularización de nuevos medios de comunicación como el cine y la radio.

En tan complicadas circunstancias, se esperaba que los dirigentes nacionales tomaran medidas que garantizaran el buen funcionamiento de la vida democrática. Sin embargo, tras el fin de la guerra se difundió cierta desconfianza en cuanto a su buena fe. Al fin y al cabo, durante el conflicto el Gobierno no había dudado en manipular a sus propios ciudadanos con imponentes campañas de propaganda. Con el cese de las hostilidades, el presidente Woodrow Wilson había puesto fin a las actividades del Committee on Public Information, y sin embargo nadie podía garantizar que análogas campañas no se repetirían - esta vez de forma disimulada- en tiempo de paz ${ }^{6}$.

Esa prevención se manifestó en la atención constante que los órganos de prensa norteamericanos dedicaron a las amenazas de la propaganda y en la realización -por parte de numerosos académicos- de abundantes estudios científicos sobre las técnicas y los efectos de la manipulación masiva. El interés por esta cuestión alentó los debates de los intelectuales sobre las dinámicas de la persuasión, las reflexiones de ilustres pensadores sobre el papel de la opinión pública en el sistema democrático y los análisis de los científicos sociales sobre el impacto de la propaganda en la población ${ }^{7}$. Este fenómeno ha sido abundantemente estudiado por distintos historiadores ${ }^{8}$. Por el contrario, han sido más bien escasos los estudios sobre el impacto que aquellos temores tuvieron en la labor de los legisladores norteamericanos. Lo cierto es que el tema de la manipulación de las masas fue central en el debate político de entonces, encontrándose en los diarios de las sesiones del Congreso incesantes referencias a las campañas propagandistas de los movimientos subversivos, de las empresas privadas o de los mismos representantes del pueblo.

El propósito del presente artículo es reconstruir la manera en la que, durante el periodo de entreguerras, el poder legislativo estadounidense reaccionó a las inquietudes relacionadas con la amenaza de la propaganda. Si en la literatura académica abundan los estudios relativos a los contenidos de la propaganda (en distintos contextos históricos y geográficos) o a los canales a través de los cuales las campañas suelen ser difundidas (prensa, panfletos, radio, cine etc.), a lo largo del presente trabajo nos centraremos en la relación entre el debate público sobre la manipulación de la ciudadanía y las medidas que tomaron los representantes del poder legislativo -tanto a nivel estatal como federal-para ofrecer una respuesta a las preocupaciones de sus electores.

6 En este periodo, el único tipo de propaganda que no pareció suscitar grandes polémicas en la sociedad estadounidense fue la que se produjo con el fin específico de ser difundida en el extranjero, por ejemplo, las campañas con las que la Division of Cultural Relations, la Office for Inter-American Affairs o la fundación Rockefeller trataban de contrastar la influencia nazi en Iberoamérica. A este propósito, véase Rospir, 2018.

7 Entre los protagonistas de estos debates destacaron sin duda Dewey, 1927; Lasswell, 1927; Lippmann, 1922; Bernays, 1923.

$8 \quad$ Collins, 2013; Gary, 1999; Marks, 1957; Migliucci, 2016; Sproule, 1997. 
La herramienta que más se utilizó para hacer frente a los peligros de la manipulación masiva de grupos radicales, empresas privadas y de los mismos aparatos gubernamentales fue la creación de numerosos comités de investigación legislativa. La Constitución estadounidense no le otorga al Congreso funciones investigativas, y sin embargo desde finales del siglo XVIII dichos comités han constituido una valiosa herramienta con la cual el poder legislativo ha podido mantener un equilibrio de fuerzas con la presidencia. El primero fue instituido en 1792 para investigar una derrota militar ante los nativos y desde entonces han sido investigados todo tipo de abusos por parte de entidades y ciudadanos extranjeros y norteamericanos, incluyéndose a los mismos representantes de las instituciones ${ }^{9}$. En total, los comités que indagaron la difusión de propaganda en el periodo de entreguerras fueron una veintena, teniendo algunos de ellos un gran impacto en el debate político de aquellos años. La mayoría fueron establecidos a nivel federal, en la Cámara de los Representantes o en el Senado de Washington D.C., otros se instituyeron a nivel estatal, por ejemplo, en los órganos legislativos de Albany (Estado de Nueva York) o de Sacramento (Estado de California).

En los primeros años después del fin de la Primera Guerra Mundial, coincidiendo con el trauma que provocó la revolución rusa y con los desórdenes provocados en ambas orillas del Atlántico por movimientos filo-bolcheviques, varias investigaciones fueron establecidas sobre la propaganda de los movimientos radicales. En la época de la Gran Depresión - un periodo marcado por el desafío político entre quienes exaltaban el libre mercado y quienes lo consideraban un elemento de inestabilidad- los legisladores dirigieron su escrutinio a las campañas de persuasión de las empresas privadas. En los años treinta los comités legislativos también centraron su atención en la propaganda de los llamados movimientos antiamericanos, en un periodo en el que las consecuencias de la crisis económica y social dispararon el número de simpatizantes de la izquierda radical, afianzándose además en Europa los regímenes totalitarios fascistas. Finalmente, fue la llegada al poder de Franklin Delano Roosevelt -un presidente que apostó por políticas intervencionistas- lo que incrementó la inquietud de algunos legisladores por la propaganda del Gobierno.

Algunos de los comités del periodo de entreguerras ya han sido objeto de estudios específicos por parte de diversos historiadores ${ }^{10}$. Se han publicado también obras académicas que, examinando un determinado periodo histórico o una problemática específica, por ejemplo, el radicalismo de finales de los años diez del siglo XX, han acabado analizando algunas de estas investigaciones legislativas ${ }^{11}$. El presente artículo examinará las principales investigaciones legislativas sobre propaganda del periodo de entreguerras. Su aportación fundamental será la reconstrucción de las motivaciones que se encontraban detrás de las acciones de los congresistas que las establecieron. La hipótesis central del trabajo es que el denominador común de estos comités fue la difusión de una fuerte sensación de desamparo ante una propaganda cuyos efectos, en aquel periodo, comenzaron a ser presentados como prodigiosos. Periodistas, académicos y políticos alertaron con asiduidad sobre las siniestras maniobras de propagandistas sin escrúpulos, creándose en el país un clima de grave ansiedad en relación con las técnicas de persuasión. Los legisladores encontraron serias

\footnotetext{
Ritchie, 2016.

Por ejemplo, los comités Lusk o Dies. Véase Pfannestiel, 2003; O’Reilly, 2014.

Jaffe, 1972.
} 
dificultades a la hora de prohibir las actividades de propaganda en un país en el que la libertad de expresión se consideraba un valor sagrado. Como veremos, casi todos los intentos de promulgar legislación restrictiva en este sentido fracasaron estrepitosamente. A la postre, lo que sí pudieron ofrecerle los legisladores a la opinión pública fue una muy mediática caza al propagandista que perduró durante todo el periodo de entreguerras. Las indagaciones legislativas de aquellos años abordaron fenómenos extremadamente desiguales, desde las campañas presidenciales a las actividades de matriz terrorista, y sin embargo la inquietud que se hallaba detrás de cada una de ella era siempre la misma.

El estudio de la actividad legislativa del periodo de entreguerras se llevará a cabo a través del análisis y contextualización de fuentes primarias recientemente recolectadas en archivos norteamericanos. Por una parte, los documentos oficiales difundidos entonces por los comités, las leyes con las que fueron instituidos y aquéllas que fueron aprobadas a partir de las sugerencias expuestas en sus informes finales, y, por la otra, los diarios de las sesiones del Congreso y los artículos publicados en la prensa de la época. El material ha sido consultado en las sedes de Washington D.C. y College Park (Maryland) de la National Archives and Records Administration, así como en los New York State Archives de Albany (Nueva York). También se han utilizado las plataformas Proquest Congressional y Hathitrust, accesibles sin limitaciones desde la Biblioteca del Congreso (Washington D.C.) y desde la biblioteca pública de Nueva York (Schwarzman Building).

\section{La propaganda radical durante la primera posguerra}

Los años que siguieron al fin de la Gran Guerra fueron extremadamente turbulentos. En Rusia las fuerzas bolcheviques acabaron asentándose en el poder, en Finlandia estalló una cruenta guerra civil, en Alemania y Hungría hubo levantamientos por parte de movimientos marxistas, produciéndose gravísimos conflictos sociales también en naciones como España (Trienio Bolchevique) o Italia (Bienio Rojo).

Sin llegar nunca a los extremos que caracterizaron la realidad de algunos países europeos, Estados Unidos también vivió, a caballo entre los años diez y veinte, momentos de extrema tensión. Las huelgas y las manifestaciones políticas y sindicales se multiplicaron y la radicalización ideológica desembocó en más de una ocasión en actos violentos, hasta el extremo de los atentados terroristas. En particular, fue la ingente propagación de doctrinas sediciosas la que acabó causando aprensión en el mundo político, consolidándose la idea de que existía un estrecho vínculo entre propaganda radical y desórdenes públicos. El Departamento de Estado recibía desde todas las esquinas del planeta informes que alertaban sobre las actividades propagandísticas que se desarrollaban en los Estados Unidos por iniciativa de extremistas extranjeros. En abril de 1921, por ejemplo, el Consulado General estadounidense en Róterdam reportaba la existencia de una oficina de prensa clandestina en Nueva York, alertándose además de la presencia de propaganda comunista en barcos que estaban navegando hacia el continente americano ${ }^{12}$.

12 Telegrama del Cónsul estadounidense en Róterdam al Secretario de Estado. Róterdam, 16-IV-1921. National Archives and Records Administration [Estados Unidos] (en adelante NARA) [College Park], RG59, General 
Por todo el país se difundió una sensación de profunda indefensión ante las distintas estrategias de manipulación. Debido a que se trataba de medios de comunicación de reciente aparición, no existía todavía una legislación específica que regulase las actividades de la radio y del cine, operando de facto dichos medios en un limbo legal ${ }^{13}$. Tras el fin del conflicto, además, las represivas leyes especiales de la época de beligerancia fueron derogadas, incluyendo aquellas relacionadas con la libertad de expresión ${ }^{14}$. Seguía existiendo la posibilidad de perseguir a los extranjeros, sin embargo, los ciudadanos norteamericanos, por radical que fuesen sus actividades, parecían intocables ${ }^{15}$. Así se lo trasmitía, en 1922, la Fiscalía General al Departamento de Estado.

While Congress has on several occasions had under consideration the passing of legislation curtailing Bolshevik propaganda in this country, the same has never become a law. At the present time, the Federal Government depends wholly upon the Deportation Statutes which empower the Secretary of Labour to deport aliens from this country who advocate, teach, believe in or distribute literature advocating the overthrow of this Government by force and violence. Citizens engaged in such activities are not liable for prosecution under any existing Federal Statutes ${ }^{16}$.

La misma sensación de impotencia, esta vez en relación con la difusión de películas con contenido ideológico subversivo, se puede percibir en una carta escrita unos años más tarde por el responsable del Bureau of Investigation, Edgar Hoover: "[The Bureau will] be unable to conduct an investigation into this matter or to instruct its Agents to inspect these films (...) This would appear to be a matter solely within the jurisdiction of the various State authorities in the location in which said films are designed to be exhibited"17.

En dicho contexto de fuerte incertidumbre, la respuesta de los legisladores fue el establecimiento de varios comités de investigación legislativa, cuya finalidad fue el análisis de las diferentes actividades de propaganda que se estaban llevando a cabo en territorio estadounidense. Un destacado antecedente se había producido durante el conflicto, cuando el Congreso investigó la propaganda de la National German-American Alliance, una federación de asociaciones de ciudadanos con orígenes germanos cuya finalidad fuera la de promover la cultura alemana en el país. A principio de 1918, un subcomité de investigación había sido establecido en la Comisión

Records of the Department of State (Declassified, State Letter, 1/11/72), Department of State Decimal File, $1910-29$, caja $7328,811.00 \mathrm{~B} / 54$.

13 Una primera regulación del sector radiofónico se llevó a cabo sólo en 1927 con la promulgación de la Radio Act. En cuanto al mundo cinematográfico, en 1930 se puso en marcha un código de autorregulación promocionado por el político republicano Will H. Hays.

14 En particular, la Sedition Act -aprobada en mayo de 1918- fue derogada en 1921. La ley prohibía el uso de "lenguaje desleal, profano, difamatorio o abusivo" contra Gobierno, bandera y fuerzas armadas.

15 Los extranjeros eran expulsados de acuerdo con las directrices de la Immigration Act (Public Law 65-221, 1918), An Act to exclude and expel from the United States aliens who are members of the anarchistic and similar classes.

16 Carta del asistente del Fiscal General al Secretario de Estado. Washington D.C., 29-IX-1922. NARA [College Park], RG59, Department of State Decimal File, 1910-29, caja 7328, 811.00B/98.

17 Carta del director del Bureau of Investigation, J. Edgar Hoover, al asistente del Secretario de Estado, W.R. Castle. Washington D.C., 1-06-1928. NARA [College Park], RG59, Department of State Decimal File, 1910-29, caja 7332, 811.00B/MotionPicture29. 
de Justicia del Senado ${ }^{18}$. Sus recomendaciones habían determinado el retiro de la Congressional Charter que se le había otorgado en $1907^{19}$.

Si durante la guerra el objetivo de los legisladores habían sido las actividades de los alemanes, durante los primeros años de paz la atención se desplazó hacia el radicalismo de izquierdas. El Comité Overman es el símbolo de esta transición. Instituido en septiembre de 1918 como subcomité de la Comisión de Justicia del Senado, en un primer momento había indagado las campañas propagandísticas de los ciudadanos alemanes y en particular aquéllas de los fabricantes de cerveza ${ }^{20}$. En febrero de 1919, sin embargo, coincidiendo con la convocatoria de una huelga general en Seattle, el Senado le encargó investigar la propaganda que los agentes bolcheviques estaban realizando en los Estados Unidos ${ }^{21}$. El comité aseguró haber demostrado la existencia de un "sistema de violencia, espionaje y distribución de literatura propagandística" por parte "del Gobierno alemán y de sus simpatizantes"22. También se señaló una correlación entre los desórdenes sociales que se estaban produciendo entonces en diversas realidades urbanas estadounidenses y la propaganda de la izquierda local, vinculándose además las acciones de esta última a los planes estratégicos del régimen bolchevique ruso.

The radical revolutionary elements in this country and the Bolshevik government of Russia have, therefore, found a common cause in support of which they can unite their forces. They are both fanning the flame of discontent and endeavoring to incite revolution. Numerous newspapers are openly advocating revolution. Literature and circular matter demanding a resort to violence are being widely circulated $^{23}$.

Estas estridentes declaraciones, de todos modos, no fueron seguidas por la presentación de pruebas concluyentes, lo que llevó al Senado a desistir de ulteriores investigaciones ${ }^{24}$. Las repercusiones de su labor, sin embargo, sí se hicieron sentir a nivel local, ya que llevaron al establecimiento de un nuevo comité en el Estado de Nueva York. En marzo de 1919, a los pocos días de finalizarse la labor del Overman, la asamblea legislativa de Albany instituyó el comité Lusk. El nexo entre los dos comités fueron las declaraciones del abogado Archibald E. Stevenson, quien -en su testimonio ante los investigadores del Overman - había lanzado una voz de alarma por la difusión de movimientos bolcheviques en Nueva York ${ }^{25}$. El equipo del senador Lusk fue más allá de la simple labor de investigación. En colaboración con la oficina del Fiscal General de los Estados Unidos, A. Mitchell Palmer, y con el Bureau of Investigation, organizó una serie de redadas contra todo movimiento sospechoso de estar vinculado con el régimen ruso, lo que resultó en cientos de personas deportadas

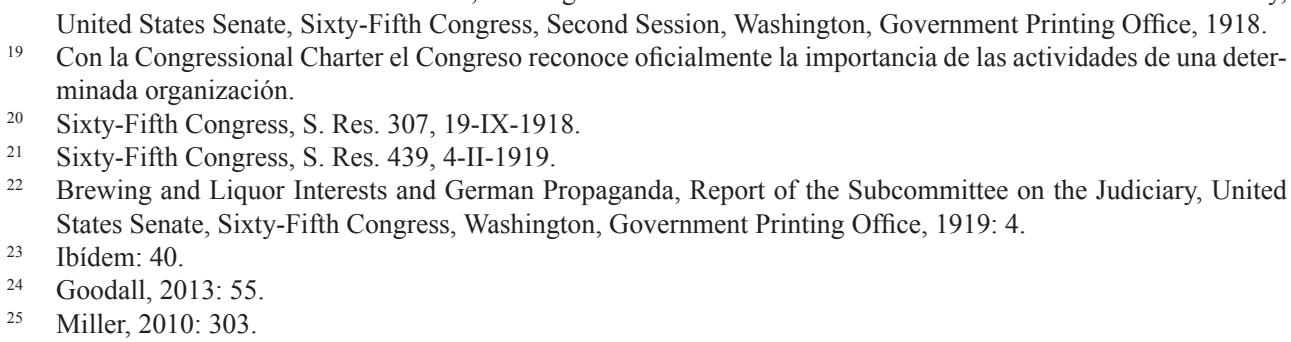


y en el secuestro de toneladas de material de propaganda ${ }^{26}$. En su informe final, el comité atribuyó "el crecimiento de movimientos radicales y revolucionarios principalmente al efecto de la propaganda" 27 . Debido a las revelaciones del comité, en el Estado se desarrolló un ambiente altamente represivo. En 1920 cinco senadores socialistas fueron expulsados de la asamblea de Albany ${ }^{28}$. El Senado local aprobó además las llamadas Lusk Laws (entre ellas, la que les exigía a los docentes un juramento de obediencia a la Constitución), que de todos modos fueron definitivamente vedadas en 1923 por el Gobernador progresista Alfred Emanuel Smith ${ }^{29}$.

Si la labor del Overman llevó a la institución del Lusk, este último llevó al establecimiento, a principios de 1920, de un subcomité -denominado Moses- por parte de la Comisión de Relaciones Exteriores del Senado federal. Los investigadores analizaron la posición de Ludwig C. A. K. Martens, un personaje que, según había revelado el comité Lusk, se declaraba representante en los Estados Unidos del régimen bolchevique ruso ${ }^{30}$. En el informe final -el documento que determinó la deportación a Rusia de Martens a principios de 1921- no se dejaban dudas sobre el hecho de que actividades de manipulación de la ciudadanía habían sido realizadas de forma reiterada ${ }^{31}$.

A principios de los años veinte, las inquietudes relacionadas con los movimientos radicales fueron disminuyendo de intensidad. El comité Moses obtuvo un espacio en la prensa considerablemente menor comparado con sus predecesores. El Congreso finalmente renunció a las investigaciones sobre propaganda subversiva, aunque ésta -por supuesto- permaneció bajo la estrecha vigilancia del Departamento de Estado y del Bureau of Investigation ${ }^{32}$. La década de los veinte fue una época de prosperidad, crecimiento y bienestar. No hubo grandes investigaciones sobre la propaganda de los movimientos radicales, pero sí que comenzó a generar cierta inquietud la propaganda de determinadas corporaciones privadas. Con el derrumbe de la bolsa de Nueva York en 1929 el miedo a las manipulaciones de los grupos empresariales alcanzó su auge.

\section{La propaganda de las grandes empresas}

Durante el periodo de entreguerras retomaron vigor unas inquietudes que habían fuertemente caracterizado el debate político norteamericano desde mediados del siglo XIX. Por una parte, el temor a que la ciudadanía pudiese ser manipulada por las campañas publicitarias dirigidas a orientar a la opinión pública a favor de los intereses de las empresas, y, por la otra, la intranquilidad ante la enorme concentración

\footnotetext{
26 Schmidt, 2000: 123-124.

27 Revolutionary radicalism: its history, purpose and tactics with an exposition and discussion of the steps being taken and required to curb it, Report of the Joint Legislative Committee Investigating Seditious Activities, Parte I, Volume II, Senado del Estado de Nueva York, Albany, J. B. Lyon, 1920: 1143.

28 Michaels, 2017: 59.

29 Heins, 2013: 29.

30 S.R. 263, 16-XII-1919, Sixty-Sixth Congress, Second Session.

31 Russian Propaganda, Report, Subcommittee of the Committee on Foreign Relations, United States Senate, Sixty-Sixth Congress, Second Session, 14-IV-1920: 4 y 15.

32 Cientos de informes sobre propaganda radical - entre ellos comunicaciones entre el Departamento y el Bureau of Investigation- pueden encontrarse en NARA [College Park], RG59, Department of State Decimal File, 191029, cajas 7327-7332, 811.00B.
} 
de poder en las manos de algunas grandes corporaciones. En las décadas que precedieron la Gran Guerra los legisladores habían realizado destacadas intervenciones para dar solución a estas problemáticas. Muy significativa fue la aprobación de la Sherman Act (1890), cuya finalidad había sido la limitación de los monopolios, o la Newspaper Publishing Act (1912), que, entre otras cosas, obligó a los periódicos a distinguir claramente entre material publicitario y contenido editorial.

La experiencia del conflicto mundial había puesto en evidencia el enorme poder de la propaganda, disparando nuevas ansiedades por el posible empleo, por parte de grandes empresas, de las estrategias de persuasión -por ejemplo, la publicación masiva en la prensa de artículos en favor de una determinada causa- que se habían ensayado con éxito durante la guerra. Dos grandes preocupaciones de la época -los lobbies y la propaganda-acabaron fusionándose en una sola gran inquietud. Ya en 1919, en el informe final del ya mencionado comité Overman, se había denunciado que los fabricantes alemanes de cerveza no habían dudado, con el fin de garantizar sus intereses económicos, en emplear grandes sumas de dinero para controlar diferentes órganos de prensa e interferir en las campañas electorales ${ }^{33}$. Gran aprensión provocaba la posibilidad de que ciertas maniobras propagandísticas se pudiesen llevar a cabo de forma oculta. A principios de 1920, el demócrata Tom D. McKeown presentó en la Cámara de los Representantes una propuesta de ley con la que pretendía que se registrasen, en el Departamento de Comercio, todas las iniciativas de recolección de dinero destinadas a la organización de campañas cuya finalidad era la de "influenciar, a través de la propaganda, la legislación nacional o la opinión pública" ${ }^{34}$. El Congresista aseguró que de esta forma se combatiría con eficacia a los grupos de presión (lobbies), a los que consideraba como una amenaza "contra la libertad y el buen Gobierno"35.

Desde mediados de los años veinte en el punto de mira de los legisladores se situaron las campañas de grandes colosos de la economía norteamericana, como las empresas que gestionaban los servicios de ferrocarril. El ex director General de la United States Railroad Administration, William G. McAdoo, había acusado a las compañías privadas de difamar, por medio de "una organizada propaganda", la gestión pública del ferrocarril, el cual -debido a la emergencia bélica- había sido administrado, desde diciembre de 1917 a marzo de 1920, por el Gobierno federal ${ }^{36}$. Las compañías privadas habían intentado convencer al contribuyente de que la gestión estatal no había sido conveniente y, en 1924, la Comisión Interestatal de Comercio les requirió reportar la cantidad de dinero invertida en la "publicación y distribución de anuncios, revistas, libros, artículos de periódicos, editoriales o panfletos" que hubiesen tenido el propósito de "crear un sentimiento público favorable" a sus intereses ${ }^{37}$.

Una acusación similar llevó, pocos años después, al establecimiento de una de las investigaciones más célebres del periodo de entreguerras. En 1928 el Senado le encargó a la Comisión Federal de Comercio una investigación sobre el empleo de propaganda por parte de empresas encargadas de administrar servicios públicos como

33 Brewing and Liquor Interests and German Propaganda, Report of the Subcommittee on the Judiciary, United States Senate, Sixty-Fifth Congress, Washington, Government Printing Office, 1919: 3-4.

34 H.R. $12378,66^{\circ}$ Congress, 6-II-1920.

35 Congressional Record, House of Representatives, 20-III-1920: 4660.

36 The New York Times, vol. LXXII, 9-IX-1923.

37 Congressional Record-Senate, 15-I-1924: 984. 
el gas y la electricidad. Una vez más a las compañías privadas se les atribuía la realización de campañas cuya finalidad era la de convencer a la opinión pública de las incuestionables ventajas de la gestión privada de los servicios públicos ${ }^{38}$. La investigación se prolongó hasta 1934, cuando fue presentado un informe final en el que se afirmaba que dichas empresas habían llevado a cabo "la más grande campaña de propaganda jamás realizada en los Estados Unidos durante la época de paz": "The record in this investigation establishes conclusively that the electric and gas utilities, since about 1919, have carried on an aggressive country-wide propaganda campaign [...] In it they have literally employed all forms of publicity except 'sky writing' [...]"39.

Entre 1929 y 1930 un subcomité de la Comisión de Asuntos Navales investigó la contratación, por parte de las grandes corporaciones estadounidenses de construcción naval, de William B. Shearer, un propagandista al que se le había encargado boicotear toda tentativa política de redimensionar el armamento de la marina ${ }^{40}$. En particular, se le acusaba de haber causado el fracaso de la Conferencia de Ginebra de 1927, cuyo objetivo había sido intentar alcanzar un acuerdo internacional para la limitación de destructores y submarinos. Muchos de los testigos que acudieron ante el comité - de acuerdo con la reconstrucción de un célebre semanario de la épocaconfirmaron estas sospechas.

[Shearer] carried on a "poisonous" anti-British propaganda. And so pervasive and influential were his activities that he was hailed in the English and European press as having been "the man who broke up the conference", a title he himself modestly does not deny ${ }^{41}$.

Unos años más tardes el poder legislativo investigó nuevamente a la industria armamentística. En 1934 el libro Merchants of Death había denunciado las siniestras maniobras con las que determinados banqueros y fabricantes de material bélico habían logrado crear, tras el estallido de la Gran Guerra en 1914, un clima favorable para la intervención norteamericana en Europa ${ }^{42}$. El escándalo causado por dicha publicación llevó al establecimiento de un comité especial en el Senado, que fue presidido por el político aislacionista Gerald Nye ${ }^{43}$. Finalmente fueron bastante escasas las pruebas relativas a la existencia de campañas de persuasión en el periodo previo a la intervención militar y sin embargo los miembros del comité insistieron en denunciar - a través de distintos medios de comunicación- los peligros de la propaganda del lobby de las armas. En un discurso radiofónico, el mismo Nye quiso alertar sobre la enorme capacidad de manipulación de dicha industria: "Americans left to their good sense and judgment will declare that never again will our country engage in war away from home. But never at any time is there let-up of that propa-

38 Gruening, 1931.

39 Utility Corporations, Summary Report of the Federal Trade Commission on Efforts by Associations and Agencies of Electric and Gas Utilities to Influence Public Opinion, Government Printing Office, Washington, 1934: 391.

40 Alleged Activities at the Geneva Conference, Hearings Before a Subcommittee of the Committee on Naval Affairs, United States Senate, Seventy-First Congress, First Session, United States Government Printing Office, Washington, 1930.

41 The Nation, Vol. 129. no 3353, 9-X-1929: 379.

42 Engelbrecht - Hanighen, 1934.

43 Munitions Industry, Report of the Special Committee on Investigation of the Munitions Industry, United States Senate, Seventy-third - Seventy-fourth Congress, Government Printing Office, Washington, 1936. 
ganda intended to convince us that other nations are more adequately prepared for war than are we" ${ }^{44}$.

A mediados de los años treinta la Administración Roosevelt lanzó un ataque frontal contra el sistema de poder de las grandes empresas. En 1935 se aprobó la National Labor Relations Act, con el que se puso límite a las medidas antisindicales de los empleadores, llevándose además a cabo -con la Public Utility Holding Company Act- una profunda regularización del sector de los servicios eléctricos. En el mismo año dos comités comenzaron a investigar las campañas que algunas grandes empresas habían realizado con el fin de presionar a los legisladores, en el intento de boicotear la aprobación de dichas normas. Uno fue presidido por el senador Hugo Black, conocido por su enemistad hacia los grandes poderes económicos y el otro por el congresista John J. O'Connor, un político que había mostrado cierta hostilidad hacia determinadas iniciativas de Roosevelt ${ }^{45}$. La forma de trabajar de los dos comités fue muy desigual. Los métodos de Black fueron "muy radicales", lo que lo llevó a ser criticado tanto por la derecha como por una parte de la izquierda ${ }^{46}$. Llegó incluso a pedir la detención de testigos que no habían acudido ante su comité, aunque sí se habían presentado ante el de O'Connor ${ }^{47}$. Este último, por su parte, reconoció que los representantes de las diversas empresas habían realizado una "extensa, bien organizada y bien financiada campaña" -que incluía "telegramas, artículos, cartas, circulares, anuncios, y todas las formas de comunicación posible" - consagrada a "prevenir la promulgación de legislación adversas". Y sin embargo añadió que apenas se habían encontrado evidencias de "contactos impropios" con los legisladores ${ }^{48}$. Al finalizarse las dos investigaciones, algunas propuestas de ley para reducir las actividades de lobbies y propaganda de las empresas fueron presentadas por políticos como el mismo Black y Howard W. Smith, y sin embargo sus textos finalmente no lograron el apoyo suficiente para convertirse en ley ${ }^{49}$.

En el contexto de la Gran Depresión, uno de los objetivos prioritarios de determinados legisladores fueron las campañas propagandísticas de los especuladores. En 1938 el senador William Borah pidió la creación de un comité para investigar la oscilación de precio de determinados productos agrícolas -en particular el trigo-debido a ilícitas campañas divulgativas de empresarios sin escrúpulos ${ }^{50}$. En el mismo año, la ofensiva legislativa contra el poder de las empresas registró un nuevo capítulo, con el establecimiento, en la Comisión de Educación y Trabajo del Senado, del llamado comité La Follette, que trabajó desde 1938 a 1941. Los congresistas investigaron algunas controvertidas estrategias -empleo de espías, contratación de esquiroles, etc.con las que determinadas corporaciones violaban los derechos de los trabajadores ${ }^{51}$. Inevitablemente, el empleo de propaganda por parte de los empleadores también fue objeto de atención, una circunstancia que fue señalada por la prensa.

\footnotetext{
Congressional Record-Senate, 12-IV-1934: 6458.

Wolf - Pederson - Daynes, 2001: 64.

Hulnick, 2016: 70.

47 Investigation of Lobbying Activities, Partial Report $n^{\circ} 1272$, Special Committee to Investigate Lobbying Activities, Senate, Seventy-fourth Congress, First Session, 29-VII-1935: 2.

48 Investigation of Lobbying Activities, Report n ${ }^{\circ} 2081$, Committee on Rules, House of Representatives, Seventyfourth Congress, Second Session, 27-II-1936: 2.

49 Zeller, 1948: 240.

50 Congressional Record-Senate, 14-VI-1938: 9135.

51 Violations of Free Speech and Rights of Labor, Preliminary Report $n^{\circ} 46$, Subcommittee of the Committee on Education and Labor, United States Senate, Seventy-Fifth Congress, First Session: 2.
} 
[El comité] has been trying to show that the National Manufacturers Association raised \$750,000 for propaganda: propaganda in newspapers, on billboards, in motion pictures, on the radio, through brochures distributed to schools and colleges and by various other means ${ }^{52}$.

Los nuevos medios de comunicación también se convirtieron en una destacada fuente de preocupación. Pese a las regulaciones del sector, siguió generando alarma el predominio que mantenían en el mundo del cine y de la radio algunas grandes corporaciones. En 1941 se puso en marcha en el Congreso un comité de investigación sobre la propaganda realizada por algunos colosos cinematográficos. Se trataba de películas bélicas destinadas -según los promotores de la investigación, entre ellos otra vez el aislacionista Nye- a influenciar el "sentimiento público" para favorecer la participación norteamericana en la Segunda Guerra Mundial ${ }^{53}$. La investigación obtuvo gran espacio en la prensa, y sin embargo fue finalmente escaso su impacto sobre los contenidos de programas y largometrajes ${ }^{54}$.

\section{Viejos y nuevos enemigos: la propaganda antiamericana}

Tras los escasos resultados alcanzados por el comité Moses, durante la década de los veinte no volvieron a constituirse comités legislativos sobre las campañas divulgativas de los movimientos radicales. Cabe sin embargo señalar que la temática de la propaganda sí estuvo en el centro del debate político cuando -entre 1921 y 1924- en el Senado fue propuesta la reapertura de las relaciones comerciales y diplomáticas con Rusia ${ }^{55}$. El mismo senador Borah, principal promotor de la reconexión con el régimen de Vladímir Lenin, tuvo que reconocer que la cuestión de la propaganda había condicionado la labor de quienes habían intentado reconstruir las relaciones con Moscú ${ }^{56}$. Tal y como aseguró el periodista Louis Fischer, las actividades propagandísticas soviéticas en los Estados Unidos se habían convertido en la principal traba para la plena consecución de dicho objetivo: "The question of communist propaganda is undoubtedly the largest single obstacle to U.S. recognition of the Soviet Government. It overshadows the issue of debts. Indeed, it perhaps plays a bigger role than all other factors combined" 57 .

La época de inactividad, desde el punto de vista de las investigaciones sobre campañas radicales, terminó en 1930, cuando la Cámara de los Representantes estableció un comité para investigar la propaganda de algunos movimientos de izquierdas ${ }^{58}$. El

$52 \quad$ The Baltimore Sun, vol. 64, 5-III-1938.

53 Senate Resolution 152 (1941).

54 Moser, 2001: 733.

55 Relations with Russia, Hearing before the Committee on Foreign Relations, United States Senate, Sixty-Sixth Congress, Third Session, Washington, Government Printing Office, 1921; Recognition of Russia, Hearings Before a Subcommittee of the Committee on Foreign Relations, United States Senate, Sixty-Eighth Congress, First Session, Washington, Government Printing Office, 1924. Las relaciones comerciales se reabrieron a principios de la década, las diplomáticas sólo en 1933.

56 Carta de Borah al secretario de Estado. Washington D.C., 14-I-1924. NARA, RG59 [College Park], Department of State Decimal File, 1910-29, caja 7329, 811.00B/260.

57 Fischer, 1931.

58 Investigation of Communist Propaganda, Hearings Before a Special Committee to Investigate Communist Activities in The United States, House of Representatives, Seventy-First Congress, Third Session, Washington, Government Printing Office, 1931. 
liderazgo lo ostentó Hamilton Fish Jr., un político conocido por su intransigente fe anticomunista. Los resultados de su investigación fueron sumamente decepcionantes. No sólo no consiguió convencer a sus colegas de la seriedad de las amenazas, negándose los congresistas a promulgar legislación que hiciese frente a las campañas comunistas, sino que fue casi completamente ignorado por la mayoría de los medios de comunicación ${ }^{59}$.

En 1933, por el contrario, la llegada al poder de los nazis en Alemania consiguió despertar en la opinión pública un fuerte interés por las actividades de la derecha radical norteamericana. Lo más preocupante parecía ser la difusión de propaganda antidemocrática entre los millones de ciudadanos que tenían orígenes italianos o germanos. Tras establecerse Adolf Hitler en la Cancillería del Reich, se instituyó, en la Comisión de Inmigración y Naturalización de la Cámara de los Representantes, un comité de emergencia presidido por el congresista Samuel Dickstein. Su cometido fue realizar "una indagación no oficial sobre las actividades de propaganda de ciudadanos extranjeros en los Estados Unidos" ${ }^{\prime 60}$. En 1934 Dickstein consiguió el establecimiento en la Cámara de los Representantes de un comité especial que investigara las llamadas Un-American Activities. Bajo el liderazgo del mismo Dickstein y de John William McCormack, dicho comité trabajó hasta 1937. Uno de sus objetivos fue la "propaganda subversiva instigada por países extranjeros" ${ }^{61}$. En el punto de mira de los investigadores acabaron sobre todo los agentes del fascismo italiano y del nacionalsocialismo alemán, aunque se indagaron también las actividades comunistas e incluso una supuesta conspiración de militares y hombres de negocios -el llamado Business Plot- para realizar un golpe de Estado contra el presidente Roosevelt ${ }^{62}$.

En 1938, siempre en la Cámara de los Representantes, se estableció el llamado comité Dies, que continuó la labor de investigación sobre actividades antiamericanas ${ }^{63}$. Oficialmente el comité indagó tanto las actividades de los grupos radicales de izquierdas como las de los de derechas. Así lo sancionaba años después el mismo Dies, en un libro en el que recapitulaba su propia vida y obra.

It investigated activities of the German-American Bund, showing how Nazi propaganda was distributed, and identified the Bund's reserve force of 5,000 uniformed storm troopers. Detailed analyses were presented of Communist activities, and front organizations, Communist finances, and propaganda centers, as well as English and foreign language publications ${ }^{64}$.

Sin embargo, es indudable que, con respecto al McCormack-Dickstein, el comité Dies se volcó principalmente en la lucha contra el peligro comunista, llegando inclu-

59 Goodall, 2014: 72.

60 Nazi Propaganda Activities by Aliens in the United States, Report, Subcommittee of Immigration and Naturalization, House of Representatives, Seventy-Third Congress, Washington, Government Printing Office, 1934.

${ }_{61}$ H. Res.108, 73d Congress, Second Session, 3-I-1934.

62 Investigation of Nazi Propaganda Activities and Investigation of Certain Other Propaganda Activities, Public Hearings before the Special Committee on Un-American Activities, House of Representatives, Seventy-Third Congress, Second Session, Part I y II, Government Printing Office, Washington, 1935. En cuanto al "Business Plot", véase Marshall, 2008.

63 Investigation of Un-American Propaganda Activities in the United States, Hearings before a Special Committee on Un-American Activities, House of Representatives, Seventy-Fifth Congress, Third Session, Vol. 1, Government Printing Office, Washington, 1938.

64 Dies, 1963: 157. 
so -según algunos autores- a convertirse en una herramienta esencialmente política para minar las políticas más progresistas del New Deal ${ }^{65}$.

Los descubrimientos de los comités sobre propaganda antiamericana tuvieron un gran impacto en los debates sobre la supervivencia del sistema democrático que se originaban entonces en los distintos órganos de prensa. Los comités McCormackDickstein y Dies, además, contribuyeron de forma determinante a la creación del clima político que llevó a la aprobación de la restrictiva Smith Act (1940), que, entre otras cosas, preveía penas severas contra quienes planteaban el derrocamiento del Gobierno ${ }^{66}$.

Este mismo clima, además, fue el detonante de unas series de leyes e investigaciones que, a caballo entre los años treinta y cuarenta, marcaron el rumbo político en algunas realidades locales. En particular, bajo la lupa acabaron las actividades de docentes cuyas tendencias políticas eran consideradas incompatibles con su papel de pedagogos. En 1935 se aprobó una normativa con la que se prohibía que el sistema educativo de Washington D.C. destinase fondos públicos para el "pago de salarios de profesores que enseñasen o apoyasen" doctrinas comunistas, una medida que fue derogada menos de dos años después ${ }^{67}$. En 1936 las autoridades legislativas de Albany le dieron mandato al senador John J. McNaboe para investigar las actividades comunistas en el Estado de Nueva York, estableciéndose además en 1940 el llamado comité Rapp-Coudert ${ }^{68}$. Este último oficialmente tenía el cometido de estudiar los problemas concernientes al sistema educativo estatal, y sin embargo desde el primer momento su interés prioritario fueron los docentes que habían manifestado una ideología radical ${ }^{69}$. Huelga decir que los sindicatos y las organizaciones estudiantiles protestaron vehementemente contra sus métodos inquisitoriales.

[El comité] has developed into a witch-hunt comparable to the old McNaboe system. It has demanded the complete roster of the Teachers' Union in an attempt to draw up a blacklist and force out those progressive instructors in the City educational system ${ }^{70}$.

Ya en vísperas de la Segunda Guerra Mundial, en el Senado de Sacramento se instituyó el llamado Comité Tenney, que trabajó hasta 1949 investigando las actividades antiamericanas en el Estado de California. También en este caso una de las principales preocupaciones de los investigadores fue la propagación en las escuelas de material propagandístico elaborado por grupos políticos radicales ${ }^{71}$.

\section{La política y la propaganda de la política}

Muy controvertida, durante el periodo de entreguerras, fue también la cuestión de la creación de consenso por parte del Gobierno federal ${ }^{72}$. La experiencia propagandís-

\footnotetext{
65 O'Reilly, 2014.

66 Public law 76-670, Seventy-Sixth Congress, 28-VI-1940.

67 District of Columbia Appropriation Act, 49 Stat. 356, 14-VI-1935. El acto sería derogado con la Public Law $n^{\circ}$ 1191, 28-V-1937.

68 Cain, 2014: 120-121.

69 Legislative Methods, Practices, Procedures, Final Report of the Joint Legislative Committee on the State Education System, State of New York, Legislative Document nº 63, Albany, Williams Press, Inc., 1947.

70 Folleto de propaganda titulado "Defend the School System". New York, aprox. 1940. New York State Archives [Albany, Estados Unidos], caja L0260-09, 21/21, RC\#614 Miscellaneous - Throwaways, leaflets, etc.

71 Scobie, 1974: 202.

72 Migliucci, 2018b.
} 
tica de la Primera Guerra Mundial, y el empleo de aparatos masivos de control de la opinión pública por parte de los regímenes totalitarios, dejaron en la ciudadanía la siniestra impresión de que los dirigentes de algunas grandes naciones ya habían adquirido la capacidad de construir el consenso popular que necesitaban para ejecutar sus planes políticos.

El Committee on Public Information cesó sus actividades domésticas en noviembre de 1918, y sin embargo durante los primeros años de la posguerra no se aplacaron los clamores en torno a los excesos de la propaganda institucional. La contratación de agentes de prensa por parte de las distintas estructuras federales no había parado de aumentar, y los departamentos ministeriales habían llegado a producir una abrumadora cantidad de material divulgativo. El Ministerio de Agricultura, por ejemplo, fue acusado de haber convertido en muy pocos años su inofensivo boletín informativo en una formidable publicación propagandística, en la que se reivindicaba lo que el Gobierno estaba haciendo por los agricultores ${ }^{73}$. De la misma forma, duras críticas fueron dirigidas contra el Ministerio de Justicia.

The Department of Justice has also constituted itself a propaganda bureau, and has sent to newspapers and magazines of this country quantities of material designed to excite public opinion against radicals, all at the expense of the government and outside the scope of the Attorney General's duties ${ }^{74}$.

Con el paso del tiempo, prácticamente todas las agencias federales acabaron en el punto de mira de los críticos. Éstos solían ser republicanos cuando la administración era demócrata y demócratas cuando en la Casa Blanca residía un republicano. Y en los años treinta el intervencionismo del Gobierno de Roosevelt disparó las alarmas entre las mismas filas del Partido Demócrata: "Charges have been made on the floors of both the Senate and the House that such a system [censorship and propaganda] has been created and is being operated by the present Administration. These charges

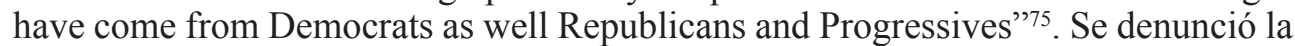
producción de películas como The River (Pare Lorentz: 1938), las periódicas charlas radiofónicas del presidente (Fireside Chats), y la creación de una estructura centralizada -la Office of Government Reports (1939) - para la coordinación de la publicidad gubernamental ${ }^{76}$.

Pese a todas las polémicas sobre los abusos propagandísticos de las agencias federales, el Congreso no instituyó ningún comité para investigar la creación de políticas públicas por parte del Gobierno. Hubo algunas destacadas propuestas -por ejemplo, la del republicano John Parnell Thomas en 1938- y sin embargo ninguna de ellas consiguió el respaldo necesario de representantes y senadores ${ }^{77}$.

De todos modos, la temática de los excesos de publicidad por parte del Gobierno sí fue tratada en distintas ocasiones por comités legislativos que, en un principio, habían sido establecidos con el fin de indagar otras cuestiones. En 1920, en el Comité Conjunto de Imprenta de la Cámara de los Representantes fue denunciada la

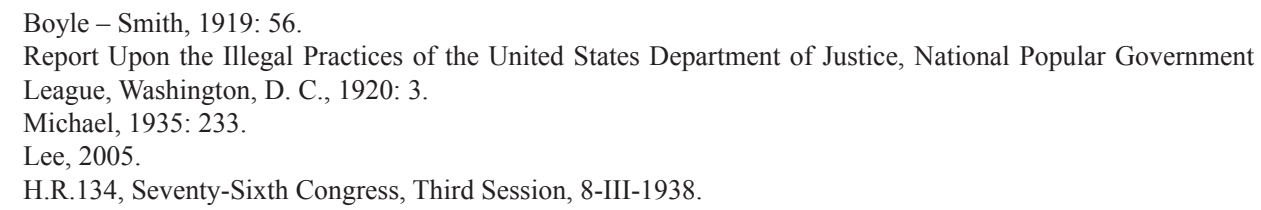


publicación, por parte de las agencias federales, de una enorme cantidad de material divulgativo, mientras que en el Comité de Privilegios y Elecciones del Senado se señalaron abusos en las actividades de publicidad realizadas durante las campañas electorales $^{78}$. El caso más célebre, sin embargo, fue el del Comité especial para investigar las agencias ejecutivas del Gobierno, que en 1936 realizó un estudio sobre las actividades de las diferentes agencias del Gobierno con el fin de mejorar su coordinación y funcionalidad ${ }^{79}$. Los investigadores revelaron irregularidades con las que los responsables de los distintos departamentos lograban evitar las normativas que limitaban el empleo de técnicas publicitarias ${ }^{80}$.

Finalmente, cabe señalar que el ya mencionado comité Dies acusó a algunas agencias del Gobierno de participar en la difusión de propaganda antiamericana. En particular, los investigadores se lanzaron al ataque de dos de las más controvertidas criaturas del New Deal, el Federal Writers Project y el Federal Theater Project. En su libro de memorias, Dies reivindicó que su investigación fue determinante para la disolución de ambos proyectos.

Several witnesses brought evidence of Communist domination in WPA's Federal Writers and Federal Theater Projects. This did not endear us to the Administration, since these enterprises had had warm support from members of the official family, including Mrs. Roosevelt. It was established by their own signatures that one-third of the members of the Federal Writers Project were Communists. Our evidence was so complete that the predominantly Liberal Congress abolished both projects $^{81}$.

\section{Conclusiones}

A lo largo del periodo de entreguerras el Congreso norteamericano investigó de forma reiterada la problemática de la propaganda en sus distintas formas, desde el proselitismo de los grupos radicales a las campañas divulgativas de los especuladores, pasando por las políticas públicas de las autoridades gubernamentales.

Tras el fin de la Primera Guerra Mundial se difundió por el país la sombría convicción de que las técnicas de manipulación de la opinión pública -eficazmente ensayadas por las naciones beligerantes durante el conflicto-ya se hallaban al alcance de personajes sin escrúpulos, lo que suponía un serio peligro para el funcionamiento -0 , incluso, para la supervivencia misma- de la democracia estadounidense. A la propaganda se le comenzaron a atribuir poderes extraordinarios, a menudo manifies-

78 Excess of governmental printed matter-president's veto of attempt to cheek waste, Extension of remarks of Hon. Albert Johnson, Appendix to the Congressional Record, 14-V-1920: 9073; A Resolution Directing the Committee on Privileges and Elections to Investigate the Campaign Expenses of Various Presidential Candidates in all Political Parties, Presidential Campaign Expenses, Hearing before a Subcommittee of the Committee on Privileges and Elections, United States Senate, Sixty-Sixth Congress, Second Session, Part 8, 1920: 1092.

79 Investigation of Executive Agencies of the Government, Report to the Select Committee to Investigate the Executive Agencies of the Government, Seventy-Fourth Congress, United States Senate, Washington, U. S. Govt. print. off., 1937.

80 Por ejemplo, la ley (38 Stat, L.208, 212, 22-X-1913) que prohibía que parte del dinero asignado para la ejecución de una norma legislativa pudiese ser destinado para publicidad (a no ser que ese tipo de gasto se especificase claramente en la ley).

81 Dies, 1963: 67. 
tamente desproporcionados. El New York Times afirmaba que habían sido "palabras, no espadas", las que habían vencido al ejército italiano en Caporetto ${ }^{82}$. George Creel, antiguo jefe del Committee on Public Information, reivindicaba el rol que habían jugado sus "proyectiles de papel" en la victoria final sobre los Imperios Centrales ${ }^{83}$. Los académicos que formaban parte del Institute for Propaganda Analysis, por su parte, se comprometieron a publicar un boletín mensual que -ante el imparable avance de las actividades de manipulación- ayudaría "a los inteligentes ciudadanos a detectar y analizar la propaganda" 84 .

Durante los años veinte y treinta, además, la problemática de la propaganda les ofreció a políticos y periodistas la posibilidad de proporcionar respuestas sencillas para problemas que ocultaban en realidad una tremenda complejidad. La conflictividad social de aquellos años fue vista como el resultado directo de las campañas divulgativas de los movimientos radicales, lo que permitía obviar un incómodo análisis sobre las injusticias y desigualdades intrínsecas al sistema socioeconómico de la época. Del mismo modo, la fuerte oposición a la regulación de los mercados se relacionaba fundamentalmente con las campañas propagandísticas de las grandes corporaciones, pasándose así por alto las resistencias que se originaban a partir de una tradición anti-intervencionista que estaba muy bien radicada en la mentalidad del pueblo norteamericano desde hacía ya muchísimo tiempo.

Y, sin embargo, si por un lado se iban trasmitiendo a la opinión pública profundas inquietudes en relación con los demoledores efectos de la manipulación de las masas, por otro resultaba extremadamente difícil perseguir a quienes se dedicaban a difundir material propagandístico. El primer lugar, en ningún momento existió consenso alrededor de lo que podía ser definido como propaganda. Los diferentes partidos se acusaban recíprocamente de manipular descaradamente a la ciudadanía, considerando sin embargo trasparentes y legítimas sus campañas informativas. En un artículo del New York Herald Tribune se aseguraba con sarcasmo que, si la propaganda del vecino era "el argumento del diablo", la propia era "la palabra de Dios" En segundo lugar, los ciudadanos norteamericanos estaban amparados por los derechos que les otorgaba la Constitución, incluyéndose entre ellos la libertad de expresión y de imprenta. Eso explica el hecho de que sólo esporádicamente se llegase a la promulgación de leyes en contra de los propagandistas, resultando evidente la incompatibilidad entre la voluntad de castigar a quienes se dedicaban a alterar la voluntad popular y la exigencia de salvaguardar aquellos derechos individuales que se hallaban en la base del sistema democrático. El comité Lusk fue uno de los pocos que consiguió que se aprobasen medidas concretas e incluso en este caso sus efectos a medio plazo fueron efimeros. Finalmente, fueron casi siempre los propagandistas extranjeros -desprotegidos en cuanto a derechos constitucionales- quienes pagaron por sus actos, con la cárcel o con la deportación.

La institución de comités de investigación legislativa sobre la problemática de la propaganda tiene que ser necesariamente explicada en el marco de esta coyuntura histórica. Por una parte, es posible que las ansiedades relacionadas con la propaganda se extendiesen a un sector importante de los legisladores, actuando seguramente

82 The New York Times, vol. 67, 31-XII-1918.

83 Creel, 1920.

${ }^{84}$ Véase por ejemplo Propaganda Analysis: A Monthly Newsletter to Help the Intelligent Citizen Detect and Analyze Propaganda, newsletter, 1, 2, Noviembre de 1937.

85 New York Herald Tribune, vol. XCIII, 8-IV-1934. 
de buena fe muchos de los investigadores que participaron en la cruzada contra la manipulación de las masas. Por el otro, resulta evidente que el establecimiento de los comités sobre propaganda respondió al imperativo de tranquilizar a una opinión pública cada vez más preocupada debido a los alarmantes relatos sobre manipulación masiva elaborados en aquellos años por políticos, periodistas e intelectuales.

Ante la imposibilidad de promulgar medidas coercitivas, la apertura de una investigación legislativa trasmitía a la población la sensación de que alguien, en lo más alto de las instituciones federales, estaba trabajando para solucionar un problema que cada vez más ciudadanos consideraban grave y urgente. Miles de personas escribían cada año a congresistas y funcionarios gubernamentales, manifestándoles su profunda sensación de inseguridad. Elocuente es, en este sentido, la respuesta que el Departamento de Estado le envió en 1939 a Geraldine Easton, una alarmada vecina de Nebraska.

With reference to your letter of October 5, 1939 to the Secretary of State requesting certain information with regard to communist activities in the United States (...) the Dies Committee is the official committee conducting investigations particularly devoted to un-American activities. You may desire to write the Superintendent of Documents, Government Printing Office, Washington, D.C., for a list of such material as may be in stock on the subjects in which you are interested ${ }^{86}$.

Algunos de estos comités se mantuvieron en actividad durante años, informando periódicamente a los ciudadanos sobre sus acciones y descubrimientos. Cuando la preocupación por una determinada problemática disminuía, los comités cerraban sus indagaciones, a menudo sin haber alcanzado ningún resultado concreto. Se creaban sin embargo nuevos comités, cuyo cometido era dar respuesta a las nuevas inquietudes del momento. Los comités recibieron miles de testimonios de gratitud por parte de ciudadanos que referían sentir sosiego gracias a su labor de investigación. Dickstein, por ejemplo, recibió numerosas cartas y telegramas por parte de ciudadanos judíos despavoridos ante la propagación a lo largo y ancho del país de doctrinas antisemitas.

I commend your proposal to investigate Nazi activities in the United States. Your great effort in this direction will surely be appreciated by All [sic.] Israel and civilized humanity. I pray to God that He bless you with strength and wisdom and power to help you safeguard the welfare of our people in this country, to serve all Israel and the community in general ${ }^{87}$.

Las investigaciones del Congreso, obviamente, no consiguieron acabar con las actividades de manipulación, como demuestra el hecho de que la envergadura de los comités sobre propaganda que estaban trabajando en vísperas de la Segunda Guerra Mundial era sin duda mayor con respecto a aquella de las indagaciones de los primeros años de paz. La propaganda de los grupos denominados antiamericanos jamás fue desactivada, y en ningún momento se halló bajo control la que realizaban las

86 Carta de Pierrepont Moffat, Jefe de la División de Asuntos Europeos, a Geraldine Easton. Washington D.C., 1-XI-1939. NARA [College Park], RG59, Department of State, Decimal File, 1930-39, caja 4725, 811.00B/1771.

87 Carta de Hillel Aronoff a Samuel Dickstein. Nueva York, 18-X-1933. NARA, RG233 [Washington D.C.], Records of the United States House of Representatives, Special Committee on Un-American Activities on Nazi Propaganda, caja 368, HR73A-F30.1. 
empresas privadas. La creación de políticas públicas de información por parte de las distintas agencias federales ni se detuvo ni tampoco disminuyó de volumen.

La labor investigadora del Congreso, sin embargo, consiguió convertir la grave sensación de impotencia de los primeros años de paz en confiado entusiasmo. Los medios de comunicación narraron con rigurosa escrupulosidad la apasionante cruzada que los comités estaban librando contra los propagandistas. Se trataba de la sempiterna lucha de los buenos contra los malos, la democracia ante las fuerzas de la opresión, el legislador honrado frente a la codicia de los poderosos. Un género, en suma, que suele ser bien recibido por el gran público. En el periodo de entreguerras, a los ciudadanos norteamericanos se les ofreció un emocionante relato: la Patria en guerra contra la manipulación de los relatos.

\section{Referencias bibliográficas}

Audoin-Rouzeau, Stéphane. “'Bourrage de crâne' et information en France en 1914-1918”. En Les sociétés européennes et la guerre de 1914-1918, editado por Becker, Jean-Jacques - Audoin-Rouzeau, Stéphane. Nanterre: Université Paris X, 1990, 163-174.

Axelrod, Alan. Selling the Great War: The Making of American Propaganda. New York: Palgrave MacMillan, 2009.

Bernays, Edward, L. Crystallizing Public Opinion. New York: Liveright, 1923.

Boyle, James E. - Smith, Guy C. "Effect of Government Control on Marketing Methods and Costs: Discussion". The American Economic Review, vol. 9, n 1 (1919), 56-60.

Cain, Timothy Reese. "Little Red Schoolhouses? Anti-Communists and Education in an 'Age of Conflicts"'. En Little 'Red Scares': Anti-Communism and Political Repression in the United States, 1921-1946, editado por Goldstein, Robert Justin. London: Routledge, 2014, 105-133.

Collins, Sue. "Propaganda Studies, The US Interwar Years". En The International Encyclopedia of Media Studies, editado por Valdivia, Angharad N. Malden: Wiley Blackwell, 2013, 578-609.

Creel, George. How We Advertised America: The First Telling of the Amazing Story of the Committee on Public Information that Carried the Gospel of Americanism to Every Corner of the Globe. New York and London: Harper \& brothers, 1920.

Dewey, John. The Public and its Problems. New York: Holt, 1927.

Dies, Martin. Martin Dies'Story. New York: Bookmailer, 1963.

Engelbrecht, Helmuth Carol - Hanighen, Frank Cleary. Merchants of Death, A Study of the International Armament Industry. London: Routledge \& Sons, 1934.

Fischer, Louis. Why recognize Russia? The Arguments for and against the Recognition of the Soviet Government by the United States. New York: J. Cape \& H. Smith, 1931.

Gary, Brett. The Nervous Liberals: Propaganda Anxieties from World War I to the Cold War. New York: Columbia Univeristy Press, 1999.

Goodall, Alex. Loyalty and Liberty: American Countersubversion from World War I to the McCarthy Era. Urbana and Chicago: University of Illinois Press, 2013.

— "Red Herrings? The Fish Committee and Anti-Communism in the Early Depression Years". En Little 'Red Scares': Anti-Communism and Political Repression in the United States, 1921-1946, editado por Goldstein, Robert Justin. London: Routledge, 2014, 71-103.

Gruening, Ernest. The Public Pays: A study of Power Propaganda. New York: Vanguard Press, 1931. 
Hanna, Martha. The Mobilization of Intellect: French Scholars and Writers during the Great War. Cambridge: Harvard University Press, 1996.

Heins, Marjorie. Priests of Our Democracy: The Supreme Court, Academic Freedom, and the Anti-Communist Purge. New York: New York University Press, 2013.

Hulnick, Blake B. "Consumer Crusade: Justice Hugo Black as Senate Investigator". Journal of Southern Legal History, vol. 24 (2016), 69-108.

Jaffe, Julian F. Crusade Against Radicalism: New York During the Red Scare, 1914-1924. Port Washington: National University Publications, 1972.

Kaminski, Joseph. "World War I and Propaganda Poster Art: Comparing the United States and German Cases". Epiphany: Journal of Transdisciplinary Studies, vol. 7, no 2 (2014), 64-81. DOI: 10.21533/epiphany.v7i2.104

Lasswell, Harold Dwight. Propaganda Technique in the World War. New York: Alfred A. Knopf, 1927.

Lee, Mordecai. The First Presidential Communications Agency: FDR's Office of Government Reports. Albany: State University of New York Press, 2005.

Lippmann, Walter. Public Opinion. New York: Harcourt, Brace and Company, 1922.

Marks, Berry Alan. The Idea of Propaganda in America. Tesis doctoral, University of Minnesota, 1957.

Marquis, Alice Goldfarb. "Words as Weapons: Propaganda in Britain and Germany during the First World War". Journal of Contemporary History, vol. 13, no 3 (1978), 467-498. DOI: $10.1177 / 002200947801300304$

Marshall, Emily Lacy. The Forgotten Treason: The Plot to Overthrow FDR. Middletown: Wesleyan University, 2008.

Messinger, Gary S. British Propaganda and the State in the First World War. Manchester: Manchester University Press, 1992.

Michael, George. Handout. New York: G.P. Putnam's Sons, 1935.

Michaels, Jonathan. McCarthyism: The Realities, Delusions and Politics Behind the 1950s Red Scare. New York: Routledge, 2017.

Migliucci, Dario. "Intolerable, peligrosa, imprescindible: intelectuales y políticos estadounidenses ante la problemática de la propaganda en el periodo de entreguerras (1919-1939)". Rubrica Contemporanea, vol. 5, no 10 (2016), 45-64. DOI: 10.5565/rev/rubrica.123

— "Opinión pública y propaganda: su definición, interpretación y significado en los Estados Unidos de la primera postguerra", Historia y Política, no 40 (2018a), 213-238. DOI: 10.18042/cepc/hp.40.08

— "Propaganda gubernamental: polémicas y (malas) prácticas en los Estados Unidos del periodo de entreguerras (1919-1941)". En Democracia y control de la opinión pública en el periodo de entreguerras, 1918-1939. Editado por Niño Rodríguez, Antonio - Rospir Zabala, Juan Ignacio. Madrid: Polifemo, 2018b, 289-334.

Miller, Kenneth E. From Progressive to New Dealer: Frederic C. Howe and American Liberalism. University Park: Pennsylvania State University Press, 2010. DOI: 10.1093/jahist/ jas 153

Mock, James R. - Larson, Cedric. Words That Won the War. The Story of the Committee on Public Information, 1917-1919. New York: Russell \& Russell, 1939.

Moser, John E. “'Gigantic Engines of Propaganda': The 1941 Senate Investigation of Hollywood". Historian, vol. 63, no 4 (2001), 731-752. DOI: 10.1111/j.1540-6563.2001. tb01943.x

O'Reilly, Kenneth. "The Dies Committee v. the New Deal: Real Americans and the Unending Search for Un-Americans". En Little 'Red Scares': Anti-Communism and Political 
Repression in the United States, 1921-1946, editado por Goldstein, Robert Justin. London: Routledge, 2014, 237-260.

Pfannestiel, Todd J. Rethinking the Red Scare: the Lusk Committee and New York's Crusade against Radicalism, 1919-1923. New York: Routledge, 2003.

Ritchie, Donald A. The U.S. Congress: A Very Short Introduction. Oxford and New York: Oxford University Press (Second Edition), 2016.

Rospir, Juan Ignacio. Comunicación de masas. Emergencia, investigación y necesidad. 'Tres memorandos de la Fundación Rockefeller (1939 y 1940)'. Madrid: Polifemo, 2018.

Row, Thomas: "Mobilizing the Nation: Italian Propaganda in the Great War". The Journal of Decorative and Propaganda Arts, vol. 24 (2002), 140-169. DOI: 10.2307/1504186

Sanders, Michael L. - Taylor, Philip M. British Propaganda during the First World War, 1914-18. Londres: MacMillan, 1982.

Schmidt, Regin. Red Scare: FBI and the Origins of Anticommunism in the United States, 1919-1943. Copenhagen: Museum Tusculanum Press, 2000.

Scobie, Ingrid Winther. “Jack B. Tenney and the 'Parasitic Menace': Anti-Communist Legislation in California 1940-1949". Pacific Historical Review, vol. 43, no 2 (1974), 188-211. DOI: $10.2307 / 3637549$

Sproule, Michael J. Propaganda and Democracy: The American Experience of Media and Mass Persuasion. Cambridge - New York: Cambridge University Press, 1997.

Vaughn, Stephen L. Holding Fast the Inner Lines. Democracy, Nationalism, and the Committee on Public Information. Chapel Hill: The University of North Carolina Press, 1980.

Wolf, Thomas Phillip - Pederson, William D. - Daynes, Byron W. (eds.). Franklin D. Roosevelt and Congress: The New Deal and its Aftermath. Armonk, N.Y.: M.E. Sharpe, 2001.

Zeller, Belle. "American Government and Politics: The Federal Regulation of Lobbying Act". The American Political Science Review, vol. 42, no 2 (1948), 239-271. DOI: $10.2307 / 1949730$ 
